\title{
NOTE ON THE RESIDUES OF THE SINGULARITIES OF A RIEMANNIAN FOLIATION
}

\author{
XIANG-MING MEI
}

\begin{abstract}
AbSTRACr. We generalize the result of Lazarov and Pasternack [3] on the residues of the singularities of a Riemannian foliation and give an explicit formula of the residues of the connected components of the singular set of a Riemannian foliation without any restriction on the dimension of the connected components of its singular set. This formula is also the generalization of the residues formula of the zero set of the Killing vector field due to Baum and Cheeger [6].
\end{abstract}

1. Introduction. Baum and Bott $[1,2]$ generalized their results on the zeros of a holomorphic vector field on a complex manifold. They studied the residues of the singularities of a holomorphic foliation, but considered only the case where the dimension of the connected components of the singular set must be equal to $r-1$, where $r$ is the dimension of the leaves. Lazarov and Pasternack [3] then studied the residues of the singularities of a Riemannian foliation defined on a Euclidean space with only one singularity at the origin. Using the same method, S. Nishikawa [4] generalized their results to the projective foliation. He also had a restriction on the dimension of the singular set of the foliation.

We consider a Riemannian foliation with singularities and give an explicit formula for the residues of the connected components of the singular set without any restriction on its dimension. The main idea of our method is to reduce the problem of computing the residues of the singular set of the foliation to the residues of a zero set of a vector field on $M$, and then use the method of transgression due to Chern and Weil.

We assume all objects appearing in this paper are $C^{\infty}$, and we discuss in real framework.

2. Riemannian foliation with singularities. Let $M$ be a compact $C^{\infty}$-manifold with $\operatorname{dim} M=n$.

Definition. 1. A $C^{\infty}$-foliation $\mathscr{F}$ of codimension $r$ defined on $M$ is a decomposition of $M$ into the union of disjoint connected subsets $\left\{L_{i}\right\}_{i \in I}$, called the leaves of $\mathscr{F}$. This decomposition requires that every point of $M$ has a neighbourhood $U$ with local coordinates $u=\left(u^{1}, \ldots, u^{n}\right): U \rightarrow R^{n}$ such that for each $L_{i}$, the components $U \cap L_{i}$ are determined by the equation $u^{n-r+1}=$ const, $\ldots, u^{n}=$ const. $(U, u)$ is called the coordinate chart. If $(U, u)$ and $(V, v)$ are two coordinate charts, then on $U \cap V$,

Received by the editors November 19, 1982.

1980 Mathematics Subject Classification. Primary 57R20; Secondary 53C12.

(C)1983 American Mathematical Society $0002-9939 / 83 \$ 1.00+\$ .25$ per page 
$d v=d u \cdot \gamma_{U V}$, where $\gamma_{U V}$ is an $(n \times n)$-matrix with entries $\left(\gamma_{U V}\right)_{i j}=\partial v^{j} / \partial u^{i}(i, j$ $=1, \ldots, n)$. Since the leaves of the foliation must be independent of the coordinate charts, the differentials $d v^{n-r+1}, \ldots, d v^{n}$ are linear combinations of the differentials $d u^{n-r+1}, \ldots, d u^{n}$. Then the matrix $\gamma_{U V}$ has the form

$$
\begin{aligned}
\gamma_{U V} & =\left(\begin{array}{c|c}
* & * \\
\hline 0 & g_{U V}
\end{array}\right), \\
d v^{i} & =\sum_{j=n-r+1}^{n} d u^{j}\left(g_{U V}\right)_{j}^{i} \quad(i=n-r+1, \ldots, n), \\
0 & =\sum_{j} d u^{j} \wedge d\left(g_{U V}\right)_{j}^{i} .
\end{aligned}
$$

It follows from the well-known Cartan lemma that $d\left(g_{U V}\right)_{j}^{i}$ is the linear combination of the $d u^{n-r+1}, \ldots, d u^{n}$.

The tangent spaces of the leaves at every point of $M$ form an integrable subbundle of the tangent bundle $T M$ of $M$, called the tangent bundle of the foliation, and denoted by $T(\mathscr{F})$. The quotient bundle $N(\mathscr{F})=T M / T(\mathscr{F})$ is called the normal bundle of the foliation. It is easily seen that $g_{U V}$ is the transition function of the bundle $N(\widetilde{F})$.

Introduce a Riemannian metric $G$ in $M$. We can consider the normal bundle $N(\widetilde{y})$ such that the fibres of $N(\widetilde{F})$ are orthogonal complements of the tangent spaces of the leaves, so the tangent bundle $T M$ of $M$ is the Whitney sum of the bundles $T(\widetilde{T})$ and $N(\widetilde{T})$.

If we choose $(U, u)$ such that the legs of the coordinate frames $\left\{\partial / \partial u^{1}, \ldots, \partial / \partial u^{n-r}\right\}$ are tangent to the leaves and the legs $\left\{\partial / \partial u^{n-r+1}, \ldots, \partial / \partial u^{n}\right\}$ are orthogonal to the leaves, then the matrix $\gamma_{U V}$ reduces to the following form:

$$
\gamma_{U V}=\left(\begin{array}{c|c}
* & 0 \\
\hline 0 & g_{U V}
\end{array}\right)
$$

and the matrix of the metric tensor in terms of these coordinate frames can be reduced to

$$
G=\left(\begin{array}{c|c}
G^{\prime} & 0 \\
\hline 0 & G^{\prime \prime}
\end{array}\right),
$$

where $G^{\prime}$ and $G^{\prime \prime}$ can be considered as the Riemannian metric of the tangent bundle $T(\widetilde{\mathscr{Y}})$ and the normal bundle $N(\widetilde{\mathscr{F}})$, respectively, induced from $G$.

A foliation $\%$ is called a Riemannian foliation if

$$
d G^{\prime \prime} \wedge d u^{n-r+1} \wedge \cdots \wedge d u^{n}=0
$$

and it means that the Riemannian metric of the normal bundle $N(\mathscr{F})$ is independent of the coordinates $u^{1}, \ldots, u^{n-r}$.

The Riemannian metric $G^{\prime \prime}$ determines uniquely a torsionless connection $\nabla^{\prime \prime}$ of the normal bundle $N(\mathscr{F})$ preserving the metric $G^{\prime \prime}$, which we call the Riemannian connection of $N(\mathscr{F})$. Let the connection matrix be $\omega^{\prime \prime}$ and the corresponding 
curvature matrix be $\Omega^{\prime \prime}=d \omega^{\prime \prime}-\omega^{\prime \prime} \wedge \omega^{\prime \prime}$. We can express the Pontrjagin characteristic form of the normal bundle $N(\mathscr{F})$ in terms of $\Omega^{\prime \prime}$, denoted by $P_{i}\left(\Omega^{\prime \prime}\right)(i=$ $1, \ldots,[r / 4])$. Then, for a Riemannian foliation, we have the following vanishing theorem:

Proposition 1 (PASTERnack [7]). Let $\varphi\left(\Omega^{\prime \prime}\right)=\tilde{\varphi}\left(P_{1}\left(\Omega^{\prime \prime}\right), \ldots, P_{[r / 4]}\left(\Omega^{\prime \prime}\right)\right)$ be the polynomial of the entries of the curvature matrix $\Omega^{\prime \prime}$ of degree 1 , where $\tilde{\varphi}$ means that $\varphi\left(\Omega^{\prime \prime}\right)$ can be expressed as another polynomial $\tilde{\varphi}$ with the arguments $P_{i}\left(\Omega^{\prime \prime}\right)(i=$ $1, \ldots,[r / 4]) ;$ then

$$
\varphi\left(\Omega^{\prime \prime}\right)=0 \text { when } 1>[r / 2] .
$$

Proof. In the coordinate chart $(U, u), \varphi\left(\Omega^{\prime \prime}\right)$ is a differential form of degree 21 in terms of $d u^{n-r+1}, \ldots, d u^{n}$, hence at least one $d u^{i}$ must be occurring in $\varphi\left(\Omega^{\prime \prime}\right)$ twice, so $\varphi\left(\Omega^{\prime \prime}\right)$ must be zero.

Now we turn to the foliation with singularities.

Definition 2. Let $\widetilde{y}$ be a $C^{\infty}$-foliation of codimension $r$ defined on $M . F_{x}$ is the fibre of the tangent bundle $T(\widetilde{F})$ of the foliation at point $x \in$ a leaf $L$. Then $F_{x}=L_{x}$ (the tangent space of $L$ at $x$ ), so $\operatorname{dim} F_{x}=\operatorname{dim} L=n-r$. If there exists some point $p \in M$ such that $\operatorname{dim} F_{x}<n-r$, then $\mathscr{F}$ is called the foliation with singularities, and $p$ is called the singular point of $\mathscr{F}$.

Let $S=U_{i} Z_{i}$ be the set of singular points of $\mathscr{F}$, where the $Z_{i}$ 's are the connected components of $S$. We assume that every $Z_{i}$ is a closed subset of $M . M$ is supposed to be compact, so the $Z_{i}$ 's are both compact and of finite number.

REMARK. There exists no leaf passing through the singular points, hence the normal bundle $N(\bar{T})$ of the foliation with singular set $S$ is defined only on $M-S$.

We can filtrate the singular set $S$ of the foliation as follows: $S=S^{1} \supset S^{2} \supset$ $\cdots \supset S^{n}{ }^{r}$, where

$$
S^{i}=\left\{x \in S: \operatorname{dim} F_{x} \leqslant n-r-i\right\} .
$$

We study only the singular set $S-S^{2}$, and denote $Z_{i} \cap\left(S-S^{2}\right)$ by $\tilde{Z}_{i}$. Since we can study the $\tilde{Z}_{i}$ 's one by one, it is convenient to omit the subscript and write $\tilde{Z}$. We further suppose that every singular point $p \in \tilde{Z}$ is regular, which means the neighbourhood of $p$ in $\tilde{Z}$ is a submanifold of $M$. We also suppose the foliation with singular set $S$ is full, which means for any open set $U$ of $M$, the vector field $X$ tangent to the leaves defined on $U \cap(M-S)$ can be extended to all $U$ such that $X(x) \in F_{x}, x \in U \cap S$.

For the full foliation with singularities, Baum $[2, \S 3]$ proved

Proposition 2. At every regular singular point $p$ of the full foliation, $F_{p} \subset S_{p}$, where $S_{p}$ is the tangent space of the singular set at $p$.

Baum also proved that in the neighbourhood of a regular singular point $p \in \tilde{Z}$, there exists a small coordinate chart $\left(U_{p}, z\right)$ such that:

Proposition 3. Let $p \in \tilde{Z}$ be a regular singular point of a full foliation $\mathcal{F}$ of codimension $r$. There exists a coordinate chart $\left(U_{p}, z\right)$ centered at $p$ and $(n-r)$ 
linearly independent vector fields $\left\{\partial / \partial z^{1}, \ldots, \partial / \partial z^{n-r-1}, X\right\}$ defined on $U_{p}$ such that

(i) For $q \in \tilde{Z} \cap U_{p},\left\{\partial(q) / \partial z^{1}, \ldots, \partial(q) / \partial z^{n-r-1}\right\} \subset \tilde{Z}_{q}$ (tangent space of $\tilde{Z}$ at $q)$.

(ii) For $x \in U_{p} \backslash \tilde{Z},\left\{\partial(x) / \partial z^{1}, \ldots, \partial(x) / \partial z^{n-r-1}, X\right\}$ form a basis of $F_{x}$.

(iii) The vector field $X=\sum_{i=n-1}^{n} a^{i}(z)\left(\partial / \partial z^{i}\right)$ is tangent to the leaves with the zero set $U_{p} \cap S$.

Proof. See $[2, \S 5]$. Note that in Baum's proof, we need not assume that $\operatorname{dim} \tilde{Z}=$ $n-r-1$.

3. Residues of the singularities. Consider a Riemannian foliation $\mathscr{F}$ of codimension $4 k-1$ with a singular set $S$ defined on $M . Z$ is a connected component of $S, \tilde{Z}=Z \cap\left(S-S^{2}\right)$. Consider the $\varepsilon$-tubular neighborhood $\tilde{Z}_{\varepsilon}$ fo $\tilde{Z}$. Let the positive real number $\varepsilon$ be sufficiently small such that $\tilde{Z}_{\varepsilon}$ can be covered by finite coordinate charts $\left(U_{p}, z\right)$ in Proposition 3. $\tilde{Z}_{\varepsilon}$ can be considered as the normal $\varepsilon$-ball bundle of $\tilde{Z}$ in $M$, and the boundary $\partial \tilde{Z}_{\varepsilon}$ of $\tilde{Z}_{\varepsilon}$ can be considered as the normal $\varepsilon$-sphere bundle of $\tilde{Z}$. Suppose $\operatorname{dim} \tilde{Z}=n-4 k+2 \nu$. Then the fibre of $\partial \tilde{Z}_{\varepsilon}$ is a $(4 k-2 \nu-1)$-dimensional space.

According to Proposition 3 and using the technique of partition of unity, it follows from the full hypothesis that there exists an open set $U$ such that $U \supset \tilde{Z}_{\varepsilon} \supset \tilde{Z}$ ( $\tilde{Z}$ is the deformation retract of $U$ ), and there exists on $U(n-4 k)$ linearly independent vector fields which can be expressed locally by $\partial / \partial z^{1}, \ldots, \partial / \partial z^{n-4 k}$ and $X=\sum_{i=n-4 k+1}^{n} a^{i}(z)\left(\partial / \partial z^{i}\right)$. Define a subbundle $E$ of fibre dimension $4 k$ of $T M$ on $U$, and let its fibre be the orthogonal complement of the vector space spanned by $\partial / \partial z^{1}, \ldots, \partial / \partial z^{n-4 k}$. Clearly the bundle $E$ is the Whitney sum of $[X]$ and $N(\mathcal{F}) \mid U$, where $[X]$ is the line bundle on $U$ generated by the vector field $X$. Let $G^{\prime}$ be the Riemannian metric of $E$ induced by $G, \nabla^{\prime}$ the associated Riemannian connection with the connection matrix $\omega^{\prime}$, and $\Omega^{\prime}=d \omega^{\prime}-\omega^{\prime} \wedge \omega^{\prime}$ the corresponding curvature matrix. Then we can construct analogously the Pontrjagin forms $P_{i}\left(\Omega^{\prime}\right)(i=1, \ldots, k)$ of $E$.

REMARK. The matrix of the metric tensol of the $E$ is

$$
G^{\prime}=\left(\begin{array}{c|c}
G(X, X) & 0 \\
\hline 0 & G^{\prime \prime}
\end{array}\right)
$$

The connection matrix of the associated Riemannian connection of $G^{\prime}$ is

$$
\omega^{\prime}=\left(\begin{array}{c|c}
\left.\omega\right|_{[X]} & 0 \\
\hline 0 & \omega^{\prime \prime}
\end{array}\right) .
$$

Let $\varphi\left(\Omega^{\prime}\right)$ be a polynomial of the entries of the curvature matrix $\Omega^{\prime}$ of degree $2 k$ such that $\varphi\left(\Omega^{\prime}\right)=\tilde{\varphi}\left(P_{1}\left(\Omega^{\prime}\right), \ldots, P_{k-1}\left(\Omega^{\prime}\right)\right)$. We define the residue of the singular set $\tilde{Z}$ associated to $\varphi\left(\Omega^{\prime}\right)$ as follows:

Definition 3. The residue $\operatorname{Res}_{\varphi}(\mathcal{F}, \tilde{Z})$ of the singular set $\tilde{Z}$ of the foliation $\mathcal{F}$ associated to the polynomial $\varphi$ of degree $2 k$ is an element of $H_{n-4 k}(\tilde{Z}, R)$ such that for a representative cycle $\tau$ of $\operatorname{Res}_{\varphi}(\mathscr{F}, \tilde{Z})$ and any closed $(n-4 k)$-form $\sigma$ of $U$ with 
support $\tilde{Z}$, we define

$$
\int_{\tau} \sigma=\int_{U} \sigma \wedge \varphi\left(\Omega^{\prime}\right)=\int_{U} \sigma \wedge \tilde{\varphi}\left(P_{1}\left(\Omega^{\prime}\right), \ldots, P_{k-1}\left(\Omega^{\prime}\right)\right) .
$$

We shall give an explicit formula for $\operatorname{Res}_{\varphi}(\mathscr{F}, \tilde{Z})$. For this reason, we wish to define another connection $\tilde{\nabla}$ of the bundle

$$
E=[X]+N(\mathscr{F}) \mid U,
$$

which is a flat extension of the Riemannian connection $\nabla^{\prime \prime}$ of the bundle $N(\mathscr{F}) \mid U$. We define the connection $\tilde{\nabla}$ such that $\tilde{\nabla}_{X}=L_{X}, \tilde{\nabla}_{Y}=\nabla_{Y}$, where $L_{X}$ is the Lie differentiation with respect to the vector field $X$ and $Y$ is the vector field orthogonal to the leaves.

Since $L_{X}(X)=0$, the connection matrix of $\tilde{\nabla}$ is

$$
\tilde{\omega}=\left(\begin{array}{c|c}
0 & 0 \\
\hline 0 & \omega^{\prime \prime}
\end{array}\right) .
$$

Let the curvature matrix of $\tilde{\omega}$ of $\tilde{\Omega}=d \tilde{\omega}-\tilde{\omega} \wedge \tilde{\omega}$. Clearly

$$
\tilde{\Omega}=\left(\begin{array}{c|c}
0 & 0 \\
\hline 0 & \Omega^{\prime \prime}
\end{array}\right),
$$

and it follows from Proposition 1 that $\varphi(\tilde{\Omega})=\varphi\left(\Omega^{\prime \prime}\right)=0$.

The difference of the connection matrices of the connections $\nabla^{\prime}$ and $\tilde{\nabla}$ of $E$ is

LEMMA 1.

$$
u=\tilde{\omega}-\omega^{\prime}=\pi \otimes\left(L_{X}-\nabla_{X}\right),
$$

where $\pi$ is a 1-form defined on $U-\tilde{Z}$ such that for any vector field $v$ on $U$, we define $\pi(v)=G(X, v) / G(X, X)$, and $\nabla_{x}=i(X) \nabla$, where $i(X)$ is the inner product with the vector field $X$.

Proof. When the vector field $Y$ is orthogonal to the leaves, then

$$
u(Y)=\tilde{\omega}(Y)-\omega^{\prime}(Y)=\omega^{\prime \prime}(Y)-\omega^{\prime \prime}(Y)=0, \quad \pi(Y)=0 ;
$$

hence the lemma holds. It remains to check for the vector field $X$ that

$$
\left(\pi \otimes\left(L_{X}-\nabla_{X}\right)\right)(X)=-\nabla_{X}(X)=-\omega(X)=-\omega^{\prime}(X)=\left(\tilde{\omega}-\omega^{\prime}\right)(X)=u(X) \text {. }
$$

Now we are in a position to state and prove our main result.

THEOREM. Let $\operatorname{dim} \tilde{Z}=n-4 k+2 \nu$ and $\tau$ be a representative cycle of $\operatorname{Res}_{\varphi}(\tilde{\mathscr{F}}, \tilde{Z})$ $\in H_{n-4 k}(\tilde{Z}, R)$. For any closed $(n-4 k)$-form $\sigma$, we have the residue formula

$$
\begin{aligned}
\int_{\tau} \sigma=\int_{U} \sigma \wedge \varphi\left(\Omega^{\prime}\right)=\int_{\tilde{Z}} \sigma \wedge\left\{\text { the coefficient of } t^{z \nu}\right. \text { in the Taylor expansion } \\
\text { of the expression } \left.\left(t \Omega^{\prime}+A_{X}\right) / E\left(t \Omega^{\perp}+A_{X}^{\perp}\right)\right\},
\end{aligned}
$$

where $A_{X}=L_{X}-\nabla_{X}, E$ is the Euler characteristic form of the normal bundle of $\tilde{Z}$ in $M, \Omega^{\perp}$ and $A_{X}^{\perp}$ are the restrictions of the curvature matrix $\Omega^{\prime}$ and the operator $A_{X}$ on the normal bundle of $\tilde{Z}$, respectively. 
Proof. Consider the homotopy $\omega_{t}$ of two connections $\tilde{\omega}$ and $\omega^{\prime}$ of the bundle $E$ on $U-\tilde{Z}$. Using Lemma 1, we have

$$
\begin{aligned}
& \omega_{t}=\omega^{\prime}+t u=\omega^{\prime}+t\left(\tilde{\omega}-\omega^{\prime}\right)=\omega^{\prime}+t \pi \otimes A_{X}, \\
& \Omega_{t}=d \omega_{t}-\omega_{t} \wedge \omega_{t}=\Omega^{\prime}+t d u-t^{2} u \wedge u-t \omega^{\prime} \wedge u .
\end{aligned}
$$

According to the Chern-Weil theory of transgression, we have

$$
\varphi(\tilde{\Omega})-\varphi\left(\Omega^{\prime}\right)=2 k \cdot d \int_{0}^{l} \varphi j(u, \underbrace{\Omega_{t}, \ldots, \Omega_{t}}_{(2 k-1)}) d t .
$$

Since $\varphi(\tilde{\Omega})=\varphi\left(\Omega^{\prime \prime}\right)=0$, then

$$
\varphi\left(\Omega^{\prime}\right)=-d \eta
$$

where $\eta$ is a $(4 k-1)$-form on $U-Z$ as follows:

$$
\begin{aligned}
& =2 k \int_{0}^{1} \varphi\left(u, \Omega_{t}, \ldots, \Omega_{t}\right) d t \\
& =2 k \int_{0}^{1} \varphi\left(\pi \otimes A_{X}, \Omega^{\prime}+d \pi \otimes A_{X}, \ldots, \Omega^{\prime}+d \pi \otimes A_{X}\right) d t \\
& =\sum_{p=0}^{2 k-1} C_{2 k}^{p} \varphi(\underbrace{\Omega^{\prime}, \ldots, \Omega^{\prime}}_{p}, \underbrace{A_{X}, \ldots, A_{X}}_{2 k-p}) \cdot \pi \wedge(d \pi)^{2 k-p-1} \\
& =\left\{\begin{array}{l}
\text { the coefficient of } t^{2 k-1} \text { in the Taylor expansion of } \\
\text { the expression } \varphi\left(t \Omega^{\prime}+A_{X}\right) \wedge \pi /(1-t d \pi) .
\end{array}\right.
\end{aligned}
$$

It follows from Definition 3 of residue that

$$
\int_{\tau} \sigma=\int_{U} \sigma \wedge \varphi\left(\Omega^{\prime}\right)=\lim _{\varepsilon \rightarrow 0} \int_{U \backslash \tilde{Z}_{\varepsilon}} \sigma \wedge \varphi\left(\Omega^{\prime}\right)=\lim _{\varepsilon \rightarrow 0} \int_{U \backslash \tilde{Z}_{\varepsilon}}-d(\sigma \wedge \eta)=\lim _{\varepsilon \rightarrow 0} \int_{\partial \tilde{Z}_{\varepsilon}} \sigma \wedge \eta
$$

(since $\sigma$ is a closed form with support $\tilde{Z} \subset U$, so $d \sigma=0$, and $\left.\sigma\right|_{\partial U}=0$ ).

$$
\begin{aligned}
\int_{\tau} \sigma=\lim _{\varepsilon \rightarrow 0} \int_{\partial \tilde{Z}_{\varepsilon}} \sigma \wedge\left\{\text { the coefficient of } t^{2 k-1}\right. \text { in the Taylor expansion } \\
\text { of the expression } \left.\varphi\left(t \Omega^{\prime}+A_{X}\right) \cdot \lim _{\varepsilon \rightarrow 0} \int_{S_{\varepsilon}} \frac{\pi}{1-t d \pi}\right\} .
\end{aligned}
$$

For the integral in parentheses, we have the following

LEMMA 2.

$$
\lim _{\varepsilon \rightarrow 0} \int_{S_{\varepsilon}} \frac{\pi}{1-t d \pi}=\frac{1}{E\left(t \Omega^{\perp}+A_{X}^{\perp}\right)} t^{2 k-2 \nu-1} .
$$

Proof. See [5, pp. 72-75].

We have

$$
\int_{\tau} \sigma=\int_{\tilde{Z}} \sigma \wedge\left\{\begin{array}{l}
\text { the coefficient of } t^{(2 k-1)-(2 k-2-1)} \text { in } \\
\text { the Taylor expansion of the expression } \\
\varphi\left(t \Omega^{\prime}+A_{X}\right) \cdot 1 / E\left(t \Omega^{\perp}+A_{X}^{\perp}\right)
\end{array}\right\},
$$

and the Theorem is proved. 
When the dimension of the singular set $\tilde{Z}$ equals the dimension of the leaves minus one, i.e. $\operatorname{dim} \tilde{Z}=n-4 k$, then $\nu=0$. In this case, we have the residue formula

$$
\operatorname{Res}_{\varphi}(\mathscr{F}, \tilde{Z})=\frac{\varphi\left(A_{X}(p)\right)}{E\left(A_{X}(p)\right)}[\tilde{Z}]=\frac{\varphi\left(A_{X}(p)\right)}{\left\{\operatorname{det}\left(A_{x}(p)\right)\right\}^{1 / 2}}[\tilde{Z}]
$$

where $p$ is any point of $\tilde{Z}$ which is the center of the coordinate chart $\left(U_{p}, z\right)$, and $[\tilde{Z}]$ is the fundamental class of $H_{n-4 k}(\tilde{Z}, R)$.

This is the analogue of the residue formula of Baum and Bott [1, Theorem 3] and Nishikawa [4, Theorem 7.1] in the case of Riemannian foliations.

4. The smooth manifold admitting a compact group action. In this section we shall give an example of our Main Theorem. Let $M$ be an $n$-dimensional compact $C^{\infty}$-manifold. A compact group $\Gamma$ acts left on $M$ smoothly and generates a $C^{\infty}$-foliation $\mathcal{F}$ on $M$. The leaves of this foliation are the orbits. Since $\Gamma$ is compact, we can introduce a $\Gamma$-invariant metric $G$ in $M$ which means the metric $G$ is independent of the position of the points in the leaves, so $\mathscr{F}$ is a Riemannian foliation. The action of $\Gamma$ is called almost free if at every point of $M$, the isotropy group of $\Gamma$ is a discrete subgroup. If at a point of $M$ the action of $\Gamma$ is not almost free, this point is called the singular point of $\mathscr{F}$. Let $S$ be the set of singular points of this foliation. It can be filtrated as follows: $S=S^{1} \supset S^{2} \supset \cdots$, where

$$
S^{i}=\{x \in S: \operatorname{dim} \text { of the isotropy group of } \Gamma \text { at } x \geqslant i\} .
$$

For the foliation generated by the left action of the compact Lie group $\Gamma$ with the singular set $S$, the results of the preceding section certainly hold.

When $\Gamma$ is a one-dimensional Lie group, the foliation is of codimension $n-1$. The leaves of $\mathscr{F}$ are the flows of the Killing vector field $X$ tangent to the leaves. In this case, our residue formula becomes

$$
\begin{aligned}
& \operatorname{Res}_{\varphi}(\mathscr{F}, \tilde{Z})=\int_{\tilde{Z}}\left\{\text { the coefficient of } t^{z \nu}\right. \text { in the Taylor expansion } \\
& \text { of the expression } \left.\left(t \Omega+A_{X}\right) / E\left(t \Omega^{\perp}+A^{X}\right)\right\} .
\end{aligned}
$$

This is the residue formula of the zero set $\tilde{Z}$ of the Killing vector field $X$ due to Baum and Cheeger [6].

If the singular set $\tilde{Z}$ involves only one point $\{p\}$, the residue formula becomes

$$
\operatorname{Res}_{\varphi}(\mathscr{F}, p)=\varphi\left(A_{X}(p)\right) /\left\{\operatorname{det}\left(A_{X}(p)\right)\right\}^{1 / 2},
$$

which is the residue formula of Lazarov and Pasternack [3].

\section{REFERENCES}

1. P. Baum and R. Bott, Singularities of holomorphic foliations, J. Differential Geom. 7 (1972), 279-342.

2. P. Baum, Structure of foliation singularities, Adv. in Math. 15 (1975), 361-374.

3. C. Lazarov and J. Pasternack, Residues and characteristic classes for Riemannian foliations, J. Differential Geom. 11 (1976), 599-612. 
4. S. Nishikawa, Residues and characteristic classes for projective foliations, Japan. J. Math. 7 (1981), 45-108.

5. S. Kobayasni, Transformation groups in differential geometn, Springer-Verlag, Berlin, 1972.

6. P. Baum and J. Cheeger, Infinitesimal isometries and Pontrjagin numbers, Topology 8 (1969), 173-193.

7. J. Pasternack, Foliation and compact Lie group action. Comment. Math. Helv 46 (1971), 467-477.

Department of Mathematics, State University of New York at Buffalo, Buffalo, New York 14216

Current address: Department of Mathematics. Beijing Teacher's College, Beijing, China 